\title{
Chlorophyll fluorescence emission spectrum inside a leaf
}

\author{
Received 19th December 2007, Accepted 6th February 2008 \\ First published as an Advance Article on the web 15th February 2008 \\ DOI: $10.1039 / b 719506 k$
}

Roberto Pedrós, ${ }^{a}$ Ismael Moya, ${ }^{b}$ Yves Goulas $^{b}$ and Stéphane Jacquemoud ${ }^{c}$

Chlorophyll $a$ fluorescence can be used as an early stress indicator. Fluorescence is also connected to photosynthesis so it can be proposed for global monitoring of vegetation status from a satellite platform. Nevertheless, the correct interpretation of fluorescence requires accurate physical models. The spectral shape of the leaf fluorescence free of any re-absorption effect plays a key role in the models and is difficult to measure. We present a vegetation fluorescence emission spectrum free of re-absorption based on a combination of measurements and modelling. The suggested spectrum takes into account the photosystem I and II spectra and their relative contribution to fluorescence. This emission spectrum is applicable to describe vegetation fluorescence in biospectroscopy and remote sensing.

\section{Introduction}

Chlorophyll $a$ fluorescence from light excited vegetation emanates in specific red and far-red spectral regions, and is produced by photosystems I and II (PSI and PSII) which are pigmentprotein complexes involved in the initial stages of photosynthesis. Generally, chlorophyll fluorescence production is inversely related to photosynthesis, except when non-photochemical quenching of fluorescence (thermal dissipation) occurs. Under stress or in moderate to high irradiance conditions, plant tissues increase heat production to dissipate excess energy. This tends to decrease fluorescence emission, at least in the initial and intermediate stages of stress. Therefore, the relative balance between the three major dissipation mechanisms - photosynthesis, heat production, and chlorophyll fluorescence emission - ultimately determines the actual pattern of response observed for fluorescence. ${ }^{1,2}$

Steady-state fluorescence, known to be highly responsive to changes in environmental conditions, is widely used as indicator of plant photosynthetic function, and can be used in the early, pre-visual detection of physiological strain. Early detection may facilitate remedial action before survival, growth and productivity are constrained, and may help to forecast long term resource quality. However, since many factors affect chlorophyll fluorescence in a similar way, this technique cannot be used to identify the presence of a particular stressor, but only to indicate apparent physiological strain.

Terrestrial vegetation systems and biosphere processes and interactions are so complex that they require detailed characterization and physically sound models. As regards biosphere dynamics, notably over short time scales, spectral reflectance and directionality of radiance alone cannot provide comprehensive information since these responses are not strictly connected to fundamental processes of plant physiology. The connection is found in vegetation fluorescence, known to convey very specific in-

\footnotetext{
${ }^{a}$ Solar Radiation Group, Department of Earth Physics and Thermodynamics, University of Valencia, Spain

${ }^{b}$ Equipe de Fluorescence, Laboratoire de Météorologie Dynamique, UMR 8359, Palaiseau, France

'Institut de Physique du Globe de Paris - Equipe de géodésie \& gravimétrie Université Paris 7 - Denis Diderot, France
}

formation regarding the efficiency of light energy usage by plants, thereby relating to plant vitality and the potential to biomass production. Therefore, the use of fluorescence has been proposed to monitor plant status from a satellite platform. ${ }^{3}$ However, there are still many unknowns regarding the measurement, analysis and exploitation of natural fluorescence, making necessary the development of appropriate models and campaigns to assess the open technical, instrumental and basic scientific issues. In this paper we present a vegetation fluorescence emission spectrum inside the leaf. This spectrum is free of re-absorption and takes into account the emission of the two photosystems involved in the initial states of photosynthesis. This emission spectrum can be used in fluorescence modelling in biospectroscopy and remote sensing. Additional radiative transfer modelling would be required to include re-absorption within the leaf.

\section{Spectral distribution of fluorescence}

The source function that fluorescence modelling requires is the product of the quantum yield of fluorescence $\varphi$ by the spectral distribution function of fluorescence emission $\eta(\lambda)$, where $\lambda$ is the wavelength of emission. The $\varphi$ values are in the range of a few percent (3-5\%), between $0 \%$ (no fluorescence) and a maximum of $10 \%$, depending on the state of the leaf photosynthetic apparatus. $\eta(\lambda)$ is termed elementary emission spectrum as it is not affected by fluorescence re-absorption within the leaf. Determining the elementary spectrum is problematic because its shape and intensity are difficult to measure experimentally. ${ }^{4,5}$ We suggest a spectrum that could be used in several models that aim to describe the fluorescence emission of leaves. ${ }^{6-9}$ One can find several publications addressing the establishment of the elementary spectrum. The SLOPE model $^{8}$ uses a fluorescence spectrum measured on beech (Fagus sylvatica L.) and elm (Ulmus minor) leaves, in which reabsorption is corrected. ${ }^{10}$ Nevertheless, the method to correct for re-absorption reported in this reference recently met with some controversy. ${ }^{11}$ The measured in vivo laser-induced chlorophyll fluorescence spectra on sunflower (Helianthus annuus L.) leaves have been parameterized mathematically as the sum of two Gaussian curves centred on 685-690 nm and 720-735 $\mathrm{nm}^{6}$ However, the spectral fluorescence distributions considered are 
already re-absorbed and do not properly represent the fluorescence elementary spectrum. Moreover a Gaussian decomposition of the emission spectrum does not rely on a physical basis.

The suggested emission spectrum is a combination of measurements and modelling in order to reduce the re-absorption effect within the leaf and obtain a result close to the elementary spectrum. The spectrum separates the contribution of the two photosystems involved in the emission spectrum of the stationary fluorescence $\left(F_{\mathrm{S}}\right)$. Furthermore, the introduction of the two photosystems, will enable one to describe the dynamics of the transitions between fluorescence spectra at $F_{0}$ (minimum of fluorescence) and $F_{\mathrm{M}}$ (maximum of fluorescence) and $F_{\mathrm{M}}$ to $F_{\mathrm{S}},{ }^{12}$ as these variations reflect different relative contributions of the two photosystems to fluorescence. Photosystems I and II are protein complexes that contain a reaction centre with its full complement of electron transfer components, as well as an array of lightharvesting (antenna) pigments. Each reaction centre type contains its own reaction centre chlorophyll, P680 in PSII and P700 in PSI. Absorbed light is transferred from a pigment molecule to another in a non-radiative transfer process called exciton transfer. Finally, the exciton either reaches the reaction centre where the chemical reactions occurs, is emitted as thermal de-excitation or as fluorescence. These three processes are in competition, which explains why photosynthesis energy conversion has to be among the fastest known chemical reactions.

At room temperature, a small fraction of the absorbed light energy is re-emitted as fluorescence. For wild type organisms, the chlorophyll $a$ fluorescence emission spectrum is characterized by a major peak at $683 \mathrm{~nm}$ (half-width of about $20 \mathrm{~nm}$ ) attributable to PSII and a broad shoulder from 700 to $750 \mathrm{~nm}$, due to both PSII and PSI, the latter to a lesser extent. ${ }^{13}$ For reasons which are unclear, even in the $F_{0}$ state where the PSII fluorescence is minimal, there is at least a minor PSI contribution to the variable fluorescence emission of chloroplasts, ${ }^{14}$ i.e. the PSI fluorescence seems to be independent of the state of its reaction centre. ${ }^{15} \mathrm{~A}$ possible explanation is that fast equilibration of excited states in PSI makes its emission spectrum independent of the excitation conditions (either direct excitation or excitation through exciton transfer from PSII to PSI or spill-over). ${ }^{16}$ So, it was usually assumed that at room temperature most of this fluorescence is emanated by PSII. ${ }^{2}$ Nevertheless, the PSI contribution is around $5 \%$ at $683 \mathrm{~nm}$ and $30-35 \%$ at the far-red shoulder. ${ }^{17,18}$ When the PSII fluorescence is maximal $\left(F_{\mathrm{M}}\right)$, the PSI contribution is $1 \%$ at $683 \mathrm{~nm}$ and $6 \%$ at the far-red shoulder. Similarly, other authors have found non-negligible PSI contribution. ${ }^{19,20}$ Therefore, even if the PSI fluorescence is small, particularly around $683 \mathrm{~nm}$, it is significant and has to be taken into account.

The determination of the PSI emission spectrum makes the isolation of PSI in eukaryotic plants without alteration necessary. Anderson and Boardman were the first to enrich PSI with their digitonin preparation D-144. ${ }^{21}$ Several attempts to purify the PSI reaction centre can also be found in the literature. ${ }^{22-24}$ However, it is not clear whether the reaction centre represents the PSI with regard to the fluorescence emission spectrum. Butler and Kitajima $^{25}$ dealt with the question by proposing a theoretical basis for the existence of the specific antenna complex closely associated with PSI. This component had the $735 \mathrm{~nm}$ fluorescence emission associated with it and was called LHC-I, indicating light-harvesting complex serving PSI. The LHC-I appears in the literature as the native PSI complex ${ }^{26}$ or intact photosystem $\mathrm{I}^{27}$

There is a line of research, and several publications, on the identification of the PSI chlorophyll proteins and the energy distribution between PSII and PSI. ${ }^{2628-35}$ Most papers include PSI fluorescence emission spectra but at low temperatures, and the reason is because there is up to 20 times more fluorescence yield compared to room temperature. ${ }^{36}$ There is also a shift in the maximum position that makes the fluorescence emission spectra at low temperature not comparable to room temperature. ${ }^{37,38}$ Very few authors show the spectra at room temperature and then only to illustrate the differences between room and low temperature where the study is focused. ${ }^{36,39,40}$ Croce and co-workers ${ }^{27}$ applied the technique developed by Bassi and Simpson ${ }^{41}$ to obtain a ratio of chlorophyll to P700 of $300: 1$, and measured the PSI fluorescence emission spectrum for eukaryotic plants at several temperatures, from room temperature to $100 \mathrm{~K}$. We have chosen the fluorescence spectrum provided at room temperature by Croce and co-workers for the following reasons. Firstly, they proved that the effect of the detergent used in the extraction causes a marked increase in the contribution of the emission band near $680 \mathrm{~nm}^{36}$ and corrected it. Secondly, the authors also found a good correlation between the measured PSI fluorescence emission spectrum and the calculated emission spectrum applying the Stepanov equation to the absorption spectrum.

Regarding PSII extraction, Berthold et al. developed a simple technique to fractionate the chloroplasts using detergent and salt treatments. ${ }^{42}$ This technique is usually called BBY and is probably the most popular way of extracting PSII from chloroplasts. The extracted PSII (usually called BBY-grana) is considered to be in native form because both the reaction centre and the lightharvesting complex (LHC-II) are present. Franck et al. produced a native PSII fluorescence emission spectrum. ${ }^{43}$ The authors measured the fluorescence spectrum for several concentrations of suspensions of isolated PSII. From the shape of the shoulder at farred wavelengths we can consider that the most similar spectrum to the native PSII form is the one with the minimum chlorophyll concentration, because it has not been re-absorbed. In Fig. 1 we present together, and with the same scale, the PSI and PSII

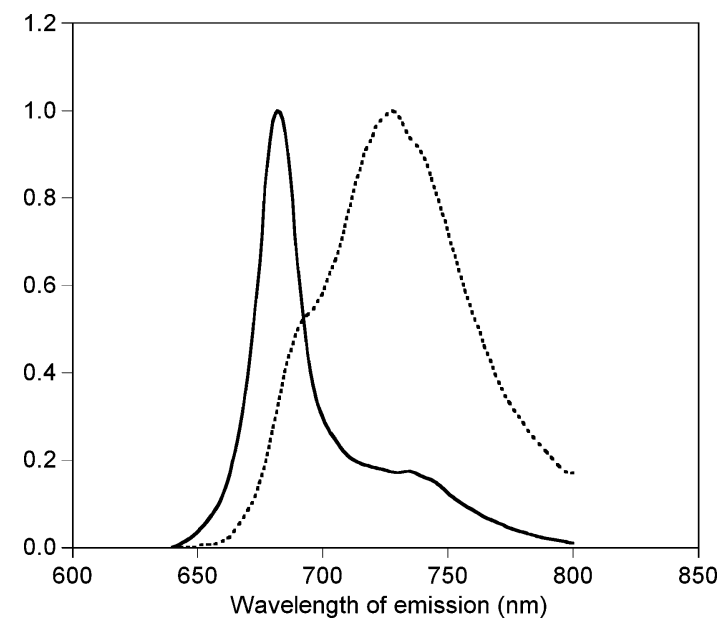

Fig. 1 PSII (straight line) and PSI (dotted line) fluorescence emission spectra in native form for plants digitalized from the figures reported in the cited papers and normalized to their maximum. 
emission spectra for plants digitalized from the figures reported in the cited papers. The PSII spectrum corresponds to chlorophyll content $0.02 \mathrm{mg} \mathrm{ml}^{-1}$. The low chlorophyll content assures that red re-absorption is minimized.

\section{PSI and PSII contribution to fluorescence}

The relative contribution of PSI and PSII is a question that has to be dealt with. Franck et al. ${ }^{43}$ proposed a relative weight for each photosystem through the $k_{\mathrm{I}}$ and $k_{\mathrm{II}}$ coefficients, respectively:

$$
\eta(\lambda)=k_{\mathrm{I}} \operatorname{PSI}(\lambda)+k_{\mathrm{II}} \operatorname{PSII}(\lambda)
$$

The authors neglected the PSI variable fluorescence due to spillover. Assuming that $k_{\mathrm{I}}=1$, they measured $k_{\mathrm{II}}=5.85$ by an indirect experimental approach, although it was suggested that $k_{\mathrm{II}}$ could be species dependent. We suggest another approach for the PSI and PSII contribution, based on the plant regulation to illumination conditions and also on the lifetime of each photosystem. Plants can cope with changes in the environment by changing the architecture of their thylakoids (grana stacking/unstacking), their stoichiometry of the reaction centres (PSII/PSI ratio), or the photosynthetic unit size. ${ }^{44-52}$ In limiting light conditions, the photosystem acclimation follows two strategies: modulation of the antenna size, and of the photosystem stoichiometry. ${ }^{46,48,51,53}$ Recently, it was shown that the regulation of antenna size is restricted to PSII while PSI-LHCI stoichiometry remains constant. $^{54}$ The general finding shows that low-light plants with large PSII antenna size have lower PSII/PSI ratios, in contrast to high-light plants with smaller PSII antenna size, which have higher PSII to PSI ratio. ${ }^{55}$ The ratio PSII/PSI is about 1.5 under many conditions, but it changes according to growth conditions. ${ }^{56}$ PSII/PSI increases with irradiance during growth, and its value is species-dependent. ${ }^{57}$ The values are in the range 1.5-1.9 for sun leaves. ${ }^{58,59}$ As an adaptive response to a change from low to high irradiance, the stoichiometry may increase after a few days. The antenna size and the PSII/PSI ratio are used as weighting factors in the combination of the photosystem emission spectra, but an additional factor is required. The fluorescence lifetime $\tau$ corresponds to the mean duration of the excited state of the chlorophyll molecules. It has been shown that the mean chlorophyll fluorescence lifetime stays almost proportional to the fluorescence yield in most experimental conditions. ${ }^{60-63} \mathrm{~A}$ direct estimation of the fluorescence quantum yield $\Phi$ can be obtained through the relation

$$
\Phi=\tau / \tau_{0}
$$

where $\tau_{0}$ is the fluorescence lifetime in the absence of any other deactivation process, with a value of $15 \mathrm{~ns}^{64}$ The two photosystems do not have the same fluorescence lifetimes, and consequently the chlorophyll fluorescence quantum yield is different. The fluorescence lifetime has a measured value of $0.1 \mathrm{~ns}$ for PSI and $0.5 \mathrm{~ns}$ for PSII. ${ }^{18,65-67}$ A weighting factor 5 is then applied to the PSII spectrum to take into account the different lifetimes. Thus, the fluorescence emission inside the leaf will have the following form

$$
\eta(\lambda)=\frac{S_{\mathrm{I}} \operatorname{PSI}(\lambda)+5 \text { Sto } S_{\mathrm{II}} \operatorname{PSII}(\lambda)}{N}
$$

where: $\operatorname{PSI}(\lambda)$ and $\operatorname{PSII}(\lambda)$ are the above mentioned emission spectra of each photosystem; $S_{\mathrm{I}}$ is the PSI antenna size; $S_{\text {II }}$ is the PSII antenna size (both sizes expressed in Chl molecules); Sto is the ratio PSII/PSI; $\lambda$ is the wavelength; and $N$ is a normalization integral so that $\eta(\lambda)$ is normalized to 1 in the range of $640-800 \mathrm{~nm}$.

Fig. 2 shows the emission spectrum in eqn (3) for measured values of photosystem stoichiometry and antenna sizes for low light ${ }^{68}$ and high light conditions. ${ }^{58}$ Both spectra present similar peak ratios due to the fact that a higher PSII/PSI ratio is balanced with a smaller PSII antenna size. However, the ratio between the two fluorescence peaks can change dramatically during induction kinetics. The changes can be explained either by a change in energy transfer, or by simple differences in the variation of fluorescence in the inner leaf tissue (with low excitation light intensity and high re-absorption) and the tissue close to the leaf surface (with high excitation light intensity and low re-absorption). Further work would be necessary in order to include induction kinetics in the emission spectra.

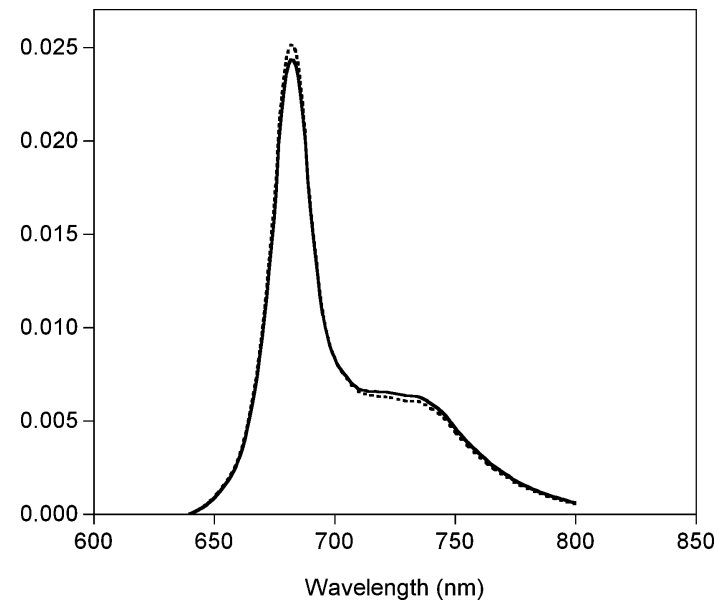

Fig. 2 Chlorophyll fluorescence emission spectrum inside a plant leaf for high light (straight line; Sto $=1.8 ; S_{\mathrm{I}}=230 ; S_{\text {II }}=250$ ) and low light conditions (dotted line; Sto $=1.47 ; S_{\mathrm{I}}=220 ; S_{\mathrm{II}}=377$ )

\section{Conclusions}

The exact spectral shape of fluorescence within the leaf free of any re-absorption effect is difficult to measure and has been derived from a combination of measurements and modelling. The proposed spectrum takes into account the contribution of each pigment-protein complex involved in the initial stages of photosynthesis, termed PSI and PSII. The PSI fluorescence emission spectrum is taken from its native form, the lightharvesting complex serving PSI. On the other hand, the PSII emission spectrum corresponds to BBY-grana, which is considered to be native as it includes both the reaction centre and the lightharvesting complex. The two spectra are combined with three different weighting factors in order to take into account the photosystem acclimatation to the environment and their different lifetimes. Firstly, the stoichiometry of PSII/PSI reaction centres is different from unity. The PSII/PSI ratio depends on light conditions during plant growth and can change as an adaptive response to changes in irradiance. Secondly, the PSII antenna size is extensively regulated according to illumination conditions. 
Thirdly, the photosystems have different lifetimes, and a weighting factor has been included. These three factors are included in the combination of the fluorescence emission of each photosystem, and the shape of the resulting spectrum is presented for high and low light conditions. The fluorescence emission spectrum inside a leaf can be useful to accurately model fluorescence in biospectroscopy and remote sensing.

\section{Acknowledgements}

This work was supported by the European Space Agency (ESTEC Contract No. 16365/02/NL/FF). The authors thank two anonymous reviewers for their suggestions to improve this manuscript.

\section{References}

1 G. H. Krause and E. Weis, Chlorophyll fluorescence and photosynthesis: the basis, Annu. Rev. Plant Physiol. Plant Mol. Biol., 1991, 42, 313-349.

2 A. J. Govindjee, Sixty-three years since Kautsky chlorophyll a fluorescence, Aust. J. Plant Physiol., 1995, 22, 131-160.

3 M. Davidson, M. Berger, I. Moya, J. Moreno, T. Laurila, M.-P. Stoll and J. Miller, Mapping photosynthesis from space-a new vegetationfluorescence technique, ESA Bull., 2003, 116, 34-37.

4 A. J. Durkin, S. Jaikumar, N. Ramanujam and R. R. Richards-Kortum, Relation between fluorescence spectra of dilute and turbid samples, Appl. Opt., 1994, 33, 414-423.

5 F. Terjung, Reabsorption of chlorophyll fluorescence and its effects on the spectral distribution and the picosecond decay of higher plant leaves, Z. Naturforsc., C, 1998, 53, 924-926.

6 N. Subhash and C. N. Mohanan, Curve-fit analysis of chlorophyll fluorescence spectra: Application to nutrient stress detection in sunflower, Remote Sensing Environ., 1997, 30, 347-356.

7 P. J. Zarco-Tejada, J. R. Miller, G. H. Mohammed and T. L. Noland, Chlorophyll fluorescence effects on vegetation apparent reflectance: I. Leaf-level measurements and model simulation, Remote Sensing Environ. L, 2000, 74, 582-595.

$8 \mathrm{~S}$. W. Maier, Modeling the Radiative Transfer in Leaves in the $300 \mathrm{~nm}$ to $2.5 \mu \mathrm{m}$ Wavelength Region taking into Consideration Chlorophyll Fluorescence - The Leaf Model SLOPE, PhD Thesis, Technische Universität München, München, 2000, 124 pp.

9 L. A. Corp, E. M. Middleton, J. E. McMurtrey, P. K. E. Campbell and L. M. Butcher, Fluorescence sensing techniques for vegetation assessment, Appl. Opt., 2006, 45, 1023-1033.

10 A. A. Gitelson, C. Buschmann and H. K. Lichtenthaler, Leaf chlorophyll fluorescence corrected for reabsorption by means of absorption and reflectance measurements, J. Plant Physiol., 1998, 152, 283-296.

11 G. B. Cordon and M. G. Lagorio, Re-absorption of chlorophyll uorescence in leaves revisited. A comparison of correction models, Photochem. Photobiol. Sci., 2006, 5, 735-740.

$12 \mathrm{G}$. Agati, Response of the in vivo chlorophyll fluorescence spectrum to environmental factors and laser excitation wavelength, Pure Appl. Opt., $1998,7,797-807$

13 D. Wong and Govindjee, Antagonistic effects of mono- and divalent cations on polarization of chlorophyll fluorescence in thylakoids and changes in excitation energy transfer, FEBS Lett., 1979, 97, 373-379.

$14 \mathrm{H}$. Dau, Molecular mechanisms and quantitative models of variable photosystem II fluorescence, Photochem. Photobiol., 1994, 60, 1-23.

15 J. M. Briantais, C. Vernotte, G. H. Krause, E. Weis, Chlorophyll $a$ fluorescence in higher plants: Chloroplasts and leaves, in Light Emission by Plants and Bacteria, ed. A. J. Govindjee and D. C. Fork, Academic Press, New York, 1986, pp. 539-583.

$16 \mathrm{H}$. W. Trissl, B. Hecks and K. Wulf, Invariable trapping times in photosystem I upon excitation of minor long-wavelength-absorbing pigments, Photochem. Photobiol., 1993, 57, 108-112.

17 T. A. Roelofs, C. H. Lee and A. R. Holzwarth, A new approach to the characterization of the primary processes in photosystem II alpha- and beta-units, Biophys. J., 1992, 61, 1147-1163.

18 G. Agati, Z. G. Cerovic and I. Moya, The effect of decreasing temperature up to chilling values on the in vivo F685/F735 chlorophyll fluorescence ratio in phaseolus vulgaris and pisum sativum: the role of the Photosystem I contribution to the $735 \mathrm{~nm}$ fluorescence band, Photochem. Photobiol., 2000, 72, 75-84.

19 E. Pfündel, Estimating the contribution of Photosystem I to total leaf chlorophyll fluorescence, Photosynth. Res., 1998, 56, 185-195.

20 R. B. Peterson, V. Oja and A. Laisk, Chlorophyll fluorescence at 680 and $730 \mathrm{~nm}$ and leaf photosynthesis, Photosynth. Res., 2001, 70, 185196.

21 J. M. Anderson and N. K. Boardman, Fractionation of the photochemical systems of photosynthesis. I. Chlorophyll contents and photochemical activities of particles isolated from spinach chloroplasts, Biochim. Biophys. Acta, 1966, 112, 403-421.

22 T. Okawa, F. Obata and K. Shibata, Two pigment proteins in spinach chloroplasts, Biochim. Biophys. Acta, 1966, 112, 223-234.

23 P. V. Sane and R. B. Park, Purification of photosystem I reaction centers from spinach stroma lamella, Biochem. Biophys. Res. Commun., 1970, 41, 206-210.

24 I. Ikegami and S. Katoh, Enrichment of photosystem I reaction center chlorophyll from spinach chloroplasts, Biochim. Biophys. Acta, 1975, 376, 588-592.

25 W. L. Butler and M. Kitajima, Energy transfer between PSII and PSI in chloroplasts, Biochim. Biophys. Acta, 1975, 396, 72-85.

26 P. Haworth, J. L. Watson and C. J. Arntzen, The detection, isolation and characterization of a light-harvesting complex, which is specifically associated with Photosystem I, Biochim. Biophys. Acta, 1983, 724, 151158.

27 R. Croce, D. Dorra, A. R. Holzwarth and R. Jennings, Fluorescence decay and spectral evolution in intact Photosystem I of higher plants, Biochemistry, 2000, 39, 6341-6348.

28 C. S. French, The distribution and action in photosynthesis of several forms of chlorophyll, Proc. Natl. Acad. Sci., USA, 1971, 68, 28932897.

29 M. Kitajima and W. L. Butler, Excitation spectra for photosystem I and photosystem II in chloroplasts and the spectral characteristics of the distribution of quanta between the two photosystems, Biochim. Biophys. Acta, 1975, 408, 297-305.

30 R. J. Strasser and W. L. Butler, Fluorescence emission spectra of photosystem I, photosystem II and the light-harvesting chlorophyll $a / b$ complex of higher plants, Biochim. Biophys. Acta, 1977, 462, 307313.

31 J. E. Mullet, J. J. Burke and J. Arntzen, Chlorophyll proteins of photosystem I, Plant Physiol., 1980, 65, 814-822.

32 R. Bassi and D. Simpson, Chlorophyll-protein complexes of barley Photosystem I, Eur. J. Biochem., 1987, 163, 221-30.

33 J. R. Evans and J. M. Anderson, Absolute absorption and relative fluorescence excitation spectra of the five major chlorophyll-protein complexes from spinach chloroplasts from spinach thylakoid membranes, Biochim. Biophys. Acta, 1987, 892, 75-82.

34 J. Knoetzel, I. Svendsen and D. J. Simpson, Identification of the photosystem I antenna polypeptides in barley. Isolation fo the three pigment-binding antenna complexes, Eur. J. Biochem., 1992, 206, 209 215.

35 J. S. S. Prakash, M. A. Baig, A. S. Bhagwat and P. Mohanty, Characterisation of senescence-induced changes in light harvesting complex II and photosystem I complex of thylakoids of Cucumis sativus cotyledons: Age induced association of LHCII with Photosystem I, J. Plant Physiol., 2003, 160, 175-184.

36 I. Mukerji and K. Sauer, Energy Transfer Dynamics of an Isolated Light Harvesting Complex of Photosystem I from Spinach: Timeresolved Fluorescence Measurements at $295 \mathrm{~K}$ and $77 \mathrm{~K}$, Biochim. Biophys. Acta, 1993, 1142, 311-320.

37 N. Murata, M. Nishimura and A. Takamiya, Fluorescence of chlorophyll in photosynthetic systems III. Emission bands of chlorophyll $a$ and the energy transfer between two pigment systems, Biochim. Biophys. Acta, 1966, 126, 234-243.

38 G. H. Krause and E. Weiss, The photosynthetic apparatus and Chlorophyll Fluorescence. An introduction, in Applications of Chlorophyll Fluorescence, ed. H. K. Lichtenthaler, Kluwer Academic Publisher, 1988, pp. 3-11.

39 I. Ikegami, Fluorescence changes related in the primary photochemical reaction in the P-700-enriched particles isolated from spinach chloroplasts, Biochim. Biophys. Acta, 1976, 449, 245-258.

40 G. Trinkunas and A. R. Holzwarth, Kinetic modeling of exciton migration in photosynthetic systems. 2. Simulations of excitation dynamics in 2-dimensional photosystem core antenna/reaction center complexes, Biophys. J., 1994, 66, 415-429. 
41 R. Bassi and D. Simpson, Chlorophyll-protein complexes of barley Photosystem I, Eur. J. Biochem., 1987, 163, 221-230.

42 D. A. Berthold, G. T. Babcock and C. F. Yocum, A highly resolved oxygen-evolving Photosystem II preparation from spinach thylakoids membranes, FEBS Lett., 1981, 134, 231-234.

43 F. Franck, P. Juneau and R. Popovic, Resolution of the photosystem I and photosystem II contributions to chlorophyll fluorescence of intact leaves at room temperature, Biochim. Biophys. Acta, 2002, 1556, 239246.

44 H. K. Lichtenthaler, C. Buschmann, M. Doll, H.-J. Fietz, T. Bach, U. Kozel and D. Meier,U. Rahmosdorf, Photosynthetic activity chloroplast ultrastructure and leaf characteristics of high-light and low-light plants and of sun and shade leaves, Photosynth. Res., 1981, 2, 115-141.

45 H. K. Lichtenthaler, G. Kuhn, U. Prenzel, C. Buschmann and D. Meier, Adaptation of chloroplast ultrastructure and of chlorophyll-protein levels to high-light and low-light growth conditions, $Z$. Naturforsc., $C$, 1982, 37, 464-475.

46 J. M. Anderson, Photoregulation of the composition, function and structure of thylakoid membranes, Annu. Rev. Plant Physiol., 1986, 37, 93-136.

47 J. M. Anderson and B. Andersson, The dynamic photosynthetic membrane and regulation of solar energy conversion, Trends Biochem. Sci., 1988, 13, 351-355.

48 A. Melis, Dynamics of photosynthetic membrane composition and function, Biochim. Biophys. Acta, 1991, 1058, 87-106.

49 J. M. Anderson, W. S. Chow and Y.-I. Park, The grand design of photosynthesis: acclimation of the photosynthetic apparatus to environmental cues, Photosynth. Res., 1995, 46, 129-139.

50 S. Malkin and D. C. Fork, Photosynthetic units of sun and shade plants, Plant Physiol., 1981, 67, 580-583.

51 W. S. Chow, A. Melis and J. M. Anderson, Adjustments of photosystem stoichiometry in chloroplasts improve the quantum efficiency of photosynthesis, Proc. Natl. Acad. Sci., USA, 1990, 87, 75027506.

52 S. Bailey, R. G. Walters, S. Jansson and P. Horton, Acclimation of Arabidopsis thaliana to the light environment: the existence of separate low-light and high-light responses, Planta, 2001, 213, 794-801.

53 T.-Y. Leong and J. M. Anderson, Adaptation of the thylakoid membranes of pea chloroplasts to light intensities. II. Regulation of electron transport capacities, electron carriers, coupling factor (CF1) activity and rates of photosynthesis, Photosynth. Res., 1984, 5, 117-128.

54 M. Ballottari, L. Dall'Osto, T. Morosinotto and R. Bassi, Contrasting behavior of higher plant photosystem I and II antenna systems during acclimation, J. Biol. Chem., 2007, 282, 8947-8958.
55 A. Akoumianaki-Ioannidou, J. H. Georgakopoulos, C. Fasseas and J. H. Argyroudi-Akoyunoglou, Photoacclimation in Spathiphyllum, J. Photochem. Photobiol., B, 2004, 73, 149-158.

56 L. Taiz, E. Zeiger, Plant Physiology, Sinauer Associates, Sunderland, Massachusetts, 1998, 2nd edn, 792 pp.

57 W. S. Chow, J. A. Anderson and A. B. Hope, Variable stoichiometries of photosystem II to photosystem I reaction centers, Photosynth. Res., 1988, 17, 277-281.

58 A. Melis, Spectroscopic Methods in Photosynthesis: Photosystem Stoichiometry and Chlorophyll Antenna Size, Philos. Trans. R. Soc. London, Ser. B, 1989, 323, 397-409.

59 D. Y. Fan, A. B. Hope and P. J. Smith, et al., The stoichiometry of the two photosystems in higher plants revisited, Biochim. Biophys. Acta, 2007, 1767, 1064-1072.

60 L. A. Tumerman and E. M. Sorokin, The photosynthetic unit: a physical or statistical model ?, Mol. Biol. USSR (English Transl.), 1967, 1, 527-535.

61 I. Moya, M. Hodges and J. C. Barbet, Modification of room temperature picosecond chlorophyll fluorescence kinetics in green algae by photosystem 2 trap closure, FEBS Lett., 1986, 198, 256-262.

$62 \mathrm{M}$. Hodges and I. Moya, Time resolved chlorophyll fluorescence studies of pigment protein complexes from photosynthetic membranes, Biochim. Biophys. Acta, 1988, 935, 41-52.

$63 \mathrm{~N}$. Moise and I. Moya, Correlation between lifetime heterogeneity and kinetics heterogeneity during chlorophyll fluorescence induction in leaves: 1. Mono-frequency phase and modulation analysis reveals a conformational change of a PSII pigment complex during the I-P thermal phase, Biochim. Biophys. Acta, 2004, 1657, 33-46.

64 S. S. Brody and E. Rabinowitch, Excitation lifetime of photosynthetic pigments in vitro and in vivo, Science, 1957, 125, 555.

65 M. Hodges and I. Moya, Modification of room temperature picosecond chlorophyll fluorescence kinetics in Photosystem 2-enriched particles by photochemistry, Biochim. Biophys. Acta, 1987, 892, 42-47.

66 A. Krieger, I. Moya and E. Weis, Energy dependent quenching of chlorophyll- $a$ fluorescence - Effect of $\mathrm{pH}$ on fluorescence induction and picosecond relaxation kinetics in thylakoid membranes and photosystem II particles, Biochim. Biophys. Acta, 1992, 1102, 167-176.

67 G. Schmuck and I. Moya, Time-resolved chlorophyll fluorescence spectra of intact leaves, Remote Sensing Environ., 1994, 47, 72-76.

68 H. Lokstein, L. Tian, J. E. W. Polle and D. DellaPenna, Xanthophyll biosynthetic mutants of Arabidopsis thaliana: altered nonphotochemical quenching of chlorophyll fluorescence is due to changes in Photosystem II antenna size and stability, Biochim. Biophys. Acta, 2002, 1553, 309-319. 